\title{
The Effects of Assymptomatic Malaria on Some Haematologi- cal Parameters among the Elderly in a Sub-Urban Community in Southern Nigeria
}

\author{
Eledo $\mathrm{BO}^{1,2^{*}}$, Tommy $\mathrm{EO}^{1}$, Onuoha $E C^{3}$, Dunga $\mathrm{KE}^{2}$ and Okamgba $\mathrm{OC}^{4}$ \\ ${ }^{1}$ Haematology Department, Federal Medical Centre, Yenagoa \\ ${ }^{2}$ Department of Medical Laboratory Science, Madonna University, Nigeria \\ ${ }^{3}$ Medical Laboratory Service, Federal University, Otuoke \\ ${ }^{4}$ Department of Medical Laboratory Science, Abia state University, Uturu, Nigeria
}

*Corresponding author: Eledo BO, Department of Medical Laboratory Science, Madonna University, Nigeria

\begin{abstract}
Malaria remains a major public health problem in endemic areas and due to neglect, the elderly ones are even more exposed now. The objective was to determine the effects (if any) of asymptomatic malaria on some hematological parameters on infected elderly subjects, identify subjects at risk of severe hematological derangement and render advice appropriately. One hundred healthy subjects (45 males and 55 females) having $\geq 60$-years-old and without clinical symptoms, were screened for malaria parasite using a rapid diagnosis test. The haematocrit (HCT), hemoglobin concentration (HB), platelet count, total white cell count (TWBC), and differential white cell count were analyzed using the Sysmex XS1000i automated hematology analyzer and all data obtained were analyzed statistically using student t-test and a p-value less than 0.05 was considered statistically significant. Out of 100 subjects, 60 subjects $(60 \%)$ were positive for malaria parasite while 40 subjects $(40 \%)$ were negative. There was significant decrease $(p<0.05)$ in the mean values of HCT $(26.1 \pm 5.8)$, HB $(9.8 \pm 1.5 \mathrm{~g} / \mathrm{dl})$ and Neutrophils $(47.5 \pm 9.5 \%)$ in positive male subjects when compared to the negative controls, HCT $(36.3 \pm 4.05 \%)$, HB $(12.5 \pm 1.20 \mathrm{~g} / \mathrm{dl})$ and Neutrophils $(49.3 \pm 9.4 \%)$ while there was a significant increase in the lymphocyte count (though within the reference range) among the positive males $(39.3 \pm 8.05 \%)$ when compared to the negative controls $(36.5 \pm 4.78 \%)$. But there was no significant difference $(P$ $>0.05)$ in the mean values of the other parameters assayed. For the female subjects, there was significant decrease $(p<0.05)$ in the mean values of Neutrophils $(45.7 \pm 11.65 \%)$ in positive subjects when compared to their negative controls (55.02 $\pm 4.38 \%)$. But there was no significant difference $(P>0.05)$ in the mean values of other parameters. This study has shown that asymptomatic malaria among the elderly has deleterious effects on some hematological parameters. Therefore, proper care should be taken by ensuring early diagnosis and treatment among them to avoid anemia and reduce risk of untimely death.
\end{abstract}

\section{Keywords}

Asymptomatic Malaria, Ageing, Hematological parameters

\section{Introduction}

The morbidity and mortality caused by malaria, especially in sub Saharan Africa is quite enormous, The disease is transmitted by female Anopheles mosquitoes which carry infective sporozoite stage of Plasmodium parasite in their salivary glands [1]. Plasmodium falciparum is responsible for about $80 \%$ of malaria infection in man and Plasmodium vivax is not seen among Africans especially West Africans due to the absence of the Duffy blood group [2]. The social and economic impact of the disease is very high. Over $90 \%$ of all deaths caused by malaria occur in sub-Saharan Africa [3]. About $25 \%$ of all estimated malaria cases in the World Health Organization African Region occur in Nigeria [3]. Malaria control strategies have focused on children under the age of 5 years and pregnant women, as the majority of malaria-related sickness and death is seen in these two groups [4]. Reports point out the considerable contribution of malaria as a cause of death in adults [5]. It is a mosquito-borne infection that killed an estimated 1.1 million people in 1998 and with an estimated 300 to 1600 million new cases, but in 2013 the disease caused an estimated 453,00 under - five deaths. According to the latest estimates, malaria mortality rates were reduced by about $47 \%$ globally and by $54 \%$ in the W.H.O. African Region between 2000 and 2013. The incidence rates declined by $30 \%$ around the world and by $34 \%$ 
in the African region. These substantial reductions occurred as a result of a major scale-up of vector control interventions, diagnostic testing and treatment with artemisinin - based combination therapies, or ACTs. The absolute numbers of malaria cases and deaths are not going down as fast as they could. The disease took an estimated 627,000 lives in 2012, mostly those of children under five years of age in Africa. Apart from children, the older people aged 60 and above have the highest prevalence rate in Nigeria [6]. According to WHO [3] statistics, malaria caused an estimated 660,000 deaths with $95 \%$ of these occurring in Africa. It is a major cause of death for about 40 percent of the world's population that live in the poorest countries [7]. A study of written narratives of a large, nationally representative, sample of all deaths [8] suggests that malaria might be a cause of about 200000 deaths per year before the age of 70 years in India. This estimate includes the 120000 deaths per year at ages 15-69 years. The number of people who die annually of malaria is roughly double the current estimate, with a huge overlooked death toll in older people. To date, there is inadequate information about the behaviour of older persons concerning management of malaria and fever, yet the elderly are vulnerable to diseases and illnesses due to their physiological state [9]. As people enter old ages it seems their health and self-definitions of old age becomes decreasingly multifaceted and increasingly related to health status [10]. Therefore a lot of changes begin to set in that may make them vulnerable to complications or more severe outcomes of infectious diseases. It is evident in this study that malaria infections were associated with complications such as cerebral involvement, renal involvement and Anemia. Previous studies have also reported similar complications among people of older age groups [11]. Respiratory disorder has also been associated with malaria in older people [12]. Early diagnosis and prompt effective treatment of malaria illness has been a cornerstone of malaria control [13].

The ageing process is associated with a progressive decline in the functional reserve of multiple organ systems, increasing the probability of dysfunction and disease. Elderly is the term used to address persons of old age. As people enter old ages, it seems their health and self-definitions of old age become decreasingly multifaceted and increasingly related to health status [10]. A lot of changes begin to set in that may make them vulnerable to complications or more severe outcomes of infectious diseases. A tentative explanation could be that the elderly are less capable of mounting an early immune response to malaria, similar to what has been observed for other infections in the elderly [14,15]. Higher parasitemia may also be a consequence of a decreased bone marrow reserve which could be aggravated by atherosclerosis, which is more prevalent in the elderly. One of the reasons why older people are not particularly thought to be vulnerable to malaria compli- cations is because it is believed that as one advances in age, partial immunity is developed against malaria. However, it is known that immune response to malaria comes along with products like cytokines involved in its pathology [16]. Furthermore the increased hormonal activities in these age groups may also be involved in severe malaria outcomes [17]. Therefore, proper attention should be given to early diagnosis and malaria prophylaxis among elderly patients.

Hematological analysis is an important aid for clinical diagnosis of systemic, infectious and some parasitic diseases. Haematopoiesis maintains adequate balance between production and destruction of blood cells. Due to the decrease in the bone marrow's ability to respond to stimuli such as bleeding, infection and cytotoxic damage during ageing this modulation becomes imbalanced. Various studies have shown that malaria infections were associated with complications such as cerebral involvement, renal involvement and anemia among people of older age groups [11]. Respiratory disorder has also been associated with malaria in older people [12]. Also, some studies suggest a decline in the stem cell population reserve, decreased sensitivity of stem cells and precursor cells to the action of cytokines, imbalance in the haematopoietic cytokine production and alterations in the microenvironment impairing homing. Anemia, generally mild, is a common problem in the elderly, especially in men. The incidence of anemia increases with age, principally after 60 -years-old. This high prevalence raised the hypothesis that lower levels of hemoglobin could be associated with the normal ageing process.

Furthermore, there is increased prevalence of geriatric non-communicable diseases in tropical malaria endemic areas particularly diabetes, hypertension etc. These diseases are associated with possible immunosuppressive functions likely to affect the integrity and sustenance of partial immunity like that developed against malaria [18]. While it is possible that impaired immunity as a result of non-communicable disease pathologies could increase susceptibility to severe infections including malaria, it is also possible that high malaria prevalence and intensity could make one susceptible to non-communicable diseases. The aetiology of these non-communicable diseases in tropical endemic areas is largely unknown [19]. However, it is known that malaria parasite activity is associated with oxidative stress in the blood and other associated tissues. Frequent exposure to the parasite without control and increased parasite intensity will only translate to oxidative stress. Increased oxidative stress on the other hand triggers inflammation, contributing significantly to the pathology of non-communicable diseases by inducing a lot of metabolic disturbances as a result of the activities of reactive oxygen species [20]. The incidence of diseases related to such metabolic disturbances for instance diabetes, renal diseases; cancers are rising and are mostly age dependent. In addition immune response to malar- 
ia is associated with the release of pro-inflammatory cytokines that are associated with the compromise of normal body metabolism that could result in non-communicable disease conditions [21]. Frequent exposure to infectious diseases such as malaria without proper management and control could have pathophysiological impacts associated with non communicable disease conditions and outcomes.

Hematological parameters such as Packed Cell Volume, Total White Blood Cell and Platelet Count are very important in the management of malaria infected patients. Samje, et al. in the University of Buea Cameroon observed $45 \%$ decreases in PCV level among malaria infected patients. [22] Attributed the decrease in PCV level (anemia) to some degree of haemolysis in malaria infected patients. The added that mild thrombocytopenia with counts down to $100 \times 109 / \mathrm{L}$ is common in malaria infected subjects. This decrease in platelet count might be due to mild splenomegaly reported in some malaria infected patients [22]. Thrombocytopenia in malaria infection was also reported in Uttarakhand State of India [23].

Furthermore, [22] showed that white cell count was slightly increased in malaria infected patients. The mean value of total white cell count among malaria infected patients was about $2 \times 109$ /L less than that of non-malaria infected elderly and this difference was statistically significant ( $p$-value $=0.0008$ ). This increase may also be due to neutrophilia reported in most cases of acute haemorrhage or due to monocytosis as seen in most cases of malaria infection. This finding is different from the work done in a Semi-Urban Community in Southwestern Nigeria where leukopenia was observed in acute malaria infection [24]. The relationship between malaria in the elderly and its outcome on their state of health in endemic areas such as Nigeria continues to be a subject of research. Hence this present work is carried out to ascertain the hematological parameters of the elderly infected with malaria parasitaemia.

\section{Materials and Methods}

\section{Research methodology}

This research is a completely randomized experimental design to estimate the effects of malaria parasitaemia on hematological parameters in the elderly in the Pilgrimage Centre Elele, including in and out-patients attending Madonna University Teaching Hospital (MUTH), Elele, Rivers State, Nigeria.

\section{Study area}

This study was carried out in the pilgrimage center Elele River State Nigeria and Madonna University Teaching Hospital (MUTH), Elele, Rivers State, Nigeria. Elele is located in the tropics of southern part of Nigeria. It is located in latitude $5^{\circ} 27-5^{\circ} 31 \mathrm{~N}$ and the longitude $6^{\circ} 57$ $7^{\circ} 85 \mathrm{E}$. The climate of the area is tropical with the mean daily temperature of $29^{\circ} \mathrm{C}$ for most of the year. The annual rainfall in this region is between 217 and $240 \mathrm{~cm}$. Its border is located within four neighboring communities namely Isiokpo, Umuagwu and Omoku; and their predominant occupation is farming and petty trading.

\section{Study population}

The population used in this study was 100 subjects comprising of 45 male and 55 female elderly subjects suspected to have malaria parasites using Care Start Malaria HRP2 (PF) rapid test kit. Those who showed negative served as the control samples. A total of 100 samples, 40 samples as control (negative for malaria), 20 control males and 20 control females. 60 samples as test (those infected with malaria), 25 males and $35 \mathrm{fe}$ males as test.

\section{Ethical clearance}

Ethical clearance was duly obtained from the ethics committee of Madonna University, Nigeria. Informed consent of the subject involved was obtained.

\section{Selective criteria for subjects}

\section{Inclusion criteria:}

- Evidence of old age which includes apparently healthy male and female above the age of 60 years.

- Only those whose blood sample showed positive for malaria parasite using Rapid malaria diagnostic kit were selected as the tests while Negative samples were selected as controls.

\section{Exclusion criteria:}

- Subjects less than 60 years were excluded from the study

- Hypertensive and Diabetic patients

- Patients with history of cardiovascular diseases

\section{Samples collection techniques}

Blood samples were obtained from the elderly subjects using standard venipuncture technique. Approximately $4 \mathrm{mls}$ of blood was collected from each subject and dispensed into sterile EDTA (Ethylenediamine Tetra Acetic Acid) bottles containing $1.5 \mathrm{mg} / \mathrm{ml}$ of blood of the anhydrous salt, mixed thoroughly to avoid clotting and then labelled.

\section{Laboratory analysis}

Hemoglobin estimation, packed cell volume, Total White cell count, Platelet count and Differential count were estimated using Sysmex XS1000i automated hematology analyzer while the malaria parasite investigation was done using rapid diagnostic test kits.

\section{Statistical analysis}

SPSS version 20 was used to carry out the statistical 
Table 1: Comparison of some haematological parameters among elderly with malaria parasites and controls.

\begin{tabular}{|c|c|c|c|}
\hline \multirow[t]{2}{*}{ Parameter } & \multicolumn{2}{|c|}{ Mean \pm Std deviation } & \multirow[t]{2}{*}{ P - Value } \\
\hline & Test $(n=60)$ & Control $(n=40)$ & \\
\hline PCV \% & $26.1 \pm 5.8$ & $36.3 \pm 4.05$ & 5.574 \\
\hline $\mathrm{HB}(\mathrm{g} / \mathrm{dl})$ & $9.8 \pm 1.5$ & $12.5 \pm 1.20$ & 0.189 \\
\hline Platelet (× 109/L) & $126.1 \pm 7.5$ & $16.6 \pm 23.4$ & 8.899 \\
\hline TWBC (× 109/L) & $6.1 \pm 3.5$ & $3.6 \pm 1.45$ & 9.421 \\
\hline NEUTROPHIL (\%) & $47.51 \pm 9.5$ & $52.5 \pm 9.4$ & 4.385 \\
\hline LYMPHOCYTE (\%) & $42.7 \pm 13.8$ & $38.3 \pm 4.5$ & 8.013 \\
\hline MONOCYTE (\%) & $5.4 \pm 0.833$ & $4.9 \pm 1.1$ & 0.991 \\
\hline EOSINOPHIL (\%) & $4.1 \pm 1.4$ & $4.0 \pm 1.2$ & 0.628 \\
\hline BASOPHIL (\%) & $0.29 \pm 0.11$ & $0.30 \pm 0.14$ & 0.931 \\
\hline
\end{tabular}

Table 2: Some haematological parameters among elderly with malaria parasites and their controls for males.

\begin{tabular}{|c|c|c|c|}
\hline \multirow[t]{2}{*}{ Parameters } & \multicolumn{2}{|c|}{ Mean \pm Std. Deviation } & \multirow[t]{2}{*}{ P -Value } \\
\hline & Test $(n=25)$ & Control $(n=20)$ & \\
\hline PCV (\%) & $30 \pm 3.21$ & $39.85 \pm 1.78$ & 0.015 \\
\hline $\mathrm{HB}(\mathrm{g} / \mathrm{dl})$ & $8.07 \pm 0.09$ & $13.13 \pm 1.02$ & 0.002 \\
\hline Platelet $\left(\times 10^{9} / \mathrm{L}\right)$ & $124.85 \pm 5.58$ & $162.9 \pm 18.94$ & 3.633 \\
\hline TWBC (× 109/L) & $7.875 \pm 0.34$ & $3.04 \pm 0.52$ & 1.163 \\
\hline Neutrophil (\%) & $50.06 \pm 7.88$ & $54.9 \pm 9.38$ & 0.039 \\
\hline Lymphocyte (\%) & $39.3 \pm 8.05$ & $36.5 \pm 4.78$ & 0.006 \\
\hline Monocyte (\%) & $5.8 \pm 0.72$ & $5.0 \pm 1.07$ & 0.973 \\
\hline Eosinophil (\%) & $4.6 \pm 1.05$ & $3.34 \pm 1.09$ & 0.454 \\
\hline Basophil (\%) & $0.24 \pm 0.09$ & $0.26 \pm 0.12$ & 0.881 \\
\hline
\end{tabular}

Table 3: Some haematological parameters among elderly with malaria parasites and their controls for females.

\begin{tabular}{|l|l|l|l|}
\hline \multirow{2}{*}{ Parameters } & \multicolumn{2}{|c|}{ Mean \pm Std. Deviation } & P - Value \\
\cline { 2 - 4 } & Test $(\mathrm{n}=25)$ & Control $(\mathrm{n}=30)$ & \\
\hline PCV $(\%)$ & $22.78 \pm 5.18$ & $32.8 \pm 2.09$ & 1.727 \\
\hline HB $(\mathrm{g} / \mathrm{dl})$ & $10.99 \pm 0.49$ & $11.95 \pm 1.10$ & 0.999 \\
\hline Platelet $\left(\times 10^{9} / \mathrm{L}\right)$ & $125.8 \pm 8.58$ & $169.7 \pm 27.38$ & 2.330 \\
\hline TWBC $\left(\times 10^{9} / \mathrm{L}\right)$ & $4.39 \pm 2.83$ & $4.23 \pm 1.82$ & 0.005 \\
\hline Neutrophil (\%) & $45.74 \pm 11.65$ & $55.02 \pm 4.38$ & 0.000 \\
\hline Lymphocyte (\%) & $43.8 \pm 9.60$ & $36.3 \pm 4.78$ & 7.424 \\
\hline Monocyte (\%) & $5.7 \pm 0.59$ & $4.3 \pm 1.15$ & 0.892 \\
\hline Eosinophil (\%) & $4.5 \pm 1.62$ & $4.1 \pm 1.32$ & 0.523 \\
\hline Basophil $(\%)$ & $0.26 \pm 0.10$ & $0.28 \pm 0.13$ & 0.883 \\
\hline
\end{tabular}

analysis. Data were presented as mean \pm standard deviation $\mathrm{T}$. test was used to show significant difference at $(p<0.05)$ among test subjects.

\section{Results and Discussion}

The Comparison of Some hematological parameters among elderly with malaria parasite and their controls were presented in Table 1. The parameters showed no significant difference $(p>0.05)$ among subjects.

Some haematological parameters among elderly males with malaria parasite and their controls were presented in Table 2. It showed that there was a significant decrease $(p<0.05)$ in the mean values of PCV, Hb and
Neutrophils, when the Test subjects were compared to their control group.

Some hematological parameters among elderly females with malaria parasite and their controls were presented in Table 3. It showed that there was a significant decrease $(p<0.05)$ in the mean values of Neutrophils when the Test subjects were compared to their control group.

\section{Discussion}

Studies have shown that the percentage of marrow space occupied by haematopoietic tissue varies throughout life, declining progressively from birth un- 
til about age 30, when it levels off. After about age 70, it again declines progressively $[25,26]$. Reported that marrow from older people can be maintained by serial transplantation in tissue culture just as long as marrow from younger people, but the number of stem cells in marrow decreases significantly with age. That Incorporation of iron in marrow culture from older people is comparable to that from younger people, but it increases less with erythropoietin stimulation. They added that healthy older animals are unable to respond to bleeding or hypoxia as efficiently as younger animals because of ineffective erythropoiesis, but whether the defect lies with the haematopoietic elements, a decrease in growth factors, or age-related architectural changes in the marrow is unclear. The decreased hemoglobin concentration and haematocrit values observed among malaria positive elderly subjects in the present study are in agreement with these findings.

Although iron uptake from the intestines is normal in the elderly, slowed erythropoiesis reduces incorporation of iron into red blood cells [26]. These changes in marrow function are not secondary to nutritional deficiencies since both total body and bone marrow iron increase with age, and both folate and vitamin B12 levels in healthy elderly people remain in the normal range. Age-related change in the peripheral blood include the following; average values of hemoglobin and haematocrit decrease slightly with age but remain within normal adult range; mean corpuscular volume increases slightly with age, but red blood cell morphologic characteristics do not change significantly, red blood cell content of 2,3-diphospho-glycerate decreases with age. RBC osmotic fragility increases with age.

The platelet count does not change with age. Increased plasma levels of two platelet granule constituents, b-thromboglobulin and platelet factor 4 , have been found in individuals over 60 years of age in comparison with younger individuals [27]. Enhanced in vitro reactivity to platelet-aggregating agents has been observed. [28] Observed decreased platelet membrane protein kinase $\mathrm{C}$ activity and translocation to the cytosol after platelet activation was noted in platelets from older subjects. Also, the non significant difference in platelet counts observed in the present study confirms these findings.

[29] Found that above age 60, the total leucocytes count tends to be lower in both sexes, primarily due to a decrease in the lymphocyte count. A decrease in the leucocytes count due to a fall in the lymphocyte and the neutrophil count was observed in women, but not in men, over age 50 . The absolute lymphocyte count was reported to be unchanged in the aged. In our study there was a significant decrease in neutrophil count among the malaria positive subjects. This is in agreement with the above findings. Also, the total white cell count obtained among the elderly subjects where slight- ly reduced, though not statistically significant ( $p>0.05)$.

This present study shows that some hematological parameters among the elderly with malaria parasites are different from those elderly not infected. This research has shown that malaria among the elderly is associated with increased anemia because their Hemoglobin estimation was less than $11 \mathrm{~g} / \mathrm{dl}$ in both sexes when compared to their control, that is to say that a lower PCV in the malaria infected elderly subjects may reflect anemia which is often mainly due to mechanical destruction of parasitized red cells as well as spleenic clearance of parasitized and defected erythrocytes. Also it is most probably that relative neutropenic leukocytopenia develops subsequently in malaria infection with a relative increase in mononuclear cells as reflected by the significantly lower neutrophils and higher lymphocytes/monocytes in the subjects. A significantly lower platelet (not statistically significant) was observed in the Test subjects. Hyper-reactive splenomegaly, especially in falciparum malaria, combined with humoral immune-response may contribute to a lower platelet count observed in the subjects in this study [30]. In the present study a significant higher level of TWBC was noted in the test subjects than the controls. This probably reflects effective immune response to malaria being a feature in endemic areas like ours [30].

Hematological parameters as an investigating tool for cases of early malaria infections may help to detect early complications associated with serious malaria infection so as to help in the care for the subjects and prevent death that may result from such complications [31].

[17] Further reported that the aged infected with Plasmodium falciparum malaria exhibited important changes in some haematological parameters with low platelet count and hemoglobin concentration being the two most important predictors of malaria infection [32,33].

\section{Conclusion}

Hematological investigation is relatively inexpensive and a less technical sophisticated way for malaria detection. Hematological parameters of malaria infected elderly subjects significantly differ from that of healthy unaffected elderly individuals. The mean values of haematological parameters of malaria infected elderly males are significantly different from those of their female counterparts. PCV, TWBC and Neutrophils are higher in the male subjects than in the females. HB, Platelets and Neutrophils are lower in the males than the females. The present study has demonstrated that asymptomatic malaria has deleterious effects on haematocrit and hemoglobin concentration which can predispose the elderly subjects to anemia. Since malaria in the elderly can lead to anemia, the use of insecticides treated nets and regular medical check-up is strongly 
advocated to reduce mortality and morbidity among the elderly.

\section{References}

1. Akinleye OT (2009) Home Management of Malaria. M.Sc Dissertation University of Lagos, Akoka, Nigeria, 5: 23-27.

2. Afolabi FE, Adenuga AO (1996) Malaria in adulthood. Nigerian Journal of General Practice 1: 9-12.

3. World Health Organisation (2010) World malaria report. Malaria Epidemiology 6: 56-55.

4. Snow RW, Guerra CA, Noor AM, Mymt HY, Hay SI (2005) The global distribution of clinical episodes of plasmodium falciparum malaria. Nature 434: 214-217.

5. World Health Organisation (2006) Malaria epidemiology. Geneva 3: 58-59.

6. World Health Organisation (2013) W.H.O global malaria programme. World Malaria Report World Health Organisation Geneva 18: 63-69.

7. World Health Organisation (2000) The abuja declaration on roll back malaria in Africa. African Heads of States and Governments Abuja, Nigeria, 56: 520-600.

8. Dhingra N, Jha P, Sharma VP, Alan A Cohen, Raju M Jotkar, et al. (2010) Adult and child malaria mortality in India: a nationally representative mortality survey. Lancet 376 : 1768-1774.

9. Asiyanbola RA (2008) Assessment of family care, housing, gender, daily activities, and physical wellbeing of the elderly in Ibadan, Nigeria. ASSET Series C 3: 63-78.

10. Breman JG, Egan A, Keusch $G$ (2001) The intolerable burden of malaria: A new look at the numbers. American Journal of Tropical Medicine and Hygiene 64: 4-7.

11. Higgias SJ, Klain KC, Liles WC (2011) Immunopathogenesis of falciparum malaria: Implications for adjunctive therapy in the management of severe and cerebral malaria. Expert Rev Anti Infect Ther 9: 803-819.

12. Lena $S$ (2011) The case for the use of oxidant antagonists as an adjunctive therapy for cerebral malaria. PPAR Research.

13. Vander HW, Presmasiri DA, Wickremasinghe AR (2005) Current trends in the control of malaria: Case management. Journal of Tropical Medicine and Public Health 29: 242-245.

14. Crossley KB, Peterson PK (1999) Infections in elderly. Clinical Infectious Diseases 22: 109-115.

15. Yoshikawa TT (1997) Perspective; Aging and Infectious Diseases; past, present and future. Journal of Infectious Diseases 176: 1053-1057.

16. Sandra Marcia Muxel, Ana Paula Freitas do Rosário, Cláudia Augusta Zago, Sheyla Inés Castillo-Méndez, Luiz Roberto Sardinha, et al. (2011) The spleen CD4 T cell response to blood stage plasmoduim chabaudi malaria develops in two phases characterized by different properties. PLos One 6: e22434.

17. Klein SL (2004) Hormonal and immunological mechanisms mediating sex differences in parasite infection. Parasite Immunology 26: 247-264.

18. Hunter DJ, Srinath R (2013) Noncommunicable Diseases. New England Journal of Medicine 369: 1336-1343.

19. Remais JV, Zeng G, Li G, Tian L, Engelgau MM (2013) Convergence of non-communicable and infectious diseas- es in low and middle income countries. Int $\mathrm{J}$ Epidemiol 42: 221-227.

20. Gottlieb MA, Morassutti AL, da Cruz IM (2011) Epidemiological transition, oxidative stress and chronic non-communicable diseases. Sciencia Medica 21: 132-141.

21. Camps J, Garcia-Heredia A (2014) Introduction: Oxidation and inflammation, a molecular link between non-communicable diseases. Adv Exp Med Biol 824: 1-4.

22. Hoffbrand A Victor D, Edward GD (2005) Postgraduate Haematology. ( $5^{\text {th }}$ edn), Massachusetts, USA: Blackwell publishing Ltd, 976, 981-985.

23. Smita C, Harish C (2013) Role of hematological parameters as an indicator of acute malaria infection in Uttarakhand State of India. Mediterranean Journal of Haematology and Infectious Diseases 5.

24. Igbeneghu C, Odaibo AB (2013) Impact of acute malaria on some haematological parameters in a semi-urban community in southwestern Nigeria. Acta Parasitologica Globalis 4: $1-5$.

25. Balducci L, Hardy C, Lyman G (2005) Haemopoiesis and aging. Journal of Cancer Treatment Resource 124: 109110.

26. Berkahn L, Keating A (2004) Haematopoiesis in the elderly. Journal of Haematology 9: 159-163.

27. Forman D, Berman A, McCabe C, Baim D (1992) PTCA in the elderly: The young-old versus the old-old. J Am Geriatr Soc 40: 19-22.

28. Nilsson B, Rolf L, Jaan H, Graciela E, Kristina N, et al. (1998) Compstatin inhibits complement and cellular activation in whole blood in two models of extracorporeal circulation. Blood 92: 661-667.

29. Cruickshank JM (1970) Some variations in the normal haemoglobin concentration. Br J Haematol 18: 523-529.

30. Bhawna S, Bharti A, Yogesh K, Aggarwal Reena (2013) Parasitemia and Hematological Alterations in Malaria: A Study from the Highly Affected Zones. Iranian Journal of Pathology 8: 1-8.

31. Ali MS, Karsani MS (2009) Haematological changes in malaria infected blood stored in blood bank refridgerator $\left(1-6^{\circ}\right.$ C). Journal of Science Technology 10: 1-7.

32. Maina RN, Walsh D, Gaddy C, Hongo G, Waitumbi J, et al. (2010) Impact of plasmodium falciparum infection on haematological parameters in adults living in Western Kenya. Malar J 9: S3-S4.

33. Sunbele IU, Theresa NA, Sarnje M, Ndzeize EM, Ndzeize EM (2010) Haematological changes and recovery associated with untreated and treated plasmodium falciparum in the mount Cameroon region. Journal of Clinical Medicine 2: 143-151. 